Check for updates

Cite this: Anal. Methods, 2017, 9, 5868

\title{
Electrochemical sensing of estradiol benzoate using hydroxyapatite with three-dimensional channel frameworks $\dagger$
}

\author{
Yu Wen, ${ }^{a}$ Jing Cui, ${ }^{a}$ Bei Shao, ${ }^{a}$ Zouting Cheng, ${ }^{a}$ Liuzheng Wang, ${ }^{a}$ Craig E. Banks (D) *b \\ and Ying Zhang (iD *a \\ Ubiquitous estradiol benzoate (17 $\beta$-estradiol-3-benzoate) in the environment and daily life has become \\ a hot issue in society since it has been proven to be closely associated with metastatic tumour \\ formation. In the current work, hydroxyapatite with three-dimensional channel frameworks has been \\ successfully demonstrated to be highly sensitive for the electroanalytical sensing of this pollutant, with \\ a detection limit of $24.4 \mathrm{ng} \mathrm{L}^{-1}(0.065 \mathrm{nM})$, attributed to the enhanced electron transport capabilities and \\ three-dimensional structure of the hydroxyapatite structure/surface.
}

Received 23rd July 2017

Accepted 17th August 2017

DOI: 10.1039/c7ay01790a

rsc.li/methods

chromatography-tandem mass spectrometry (LC-MS/MS).,15

\section{Introduction}

Endocrine disrupting chemicals (EDCs) including estrogens, triazine, phthalates, etc. have gained global attention because of their well-known serious adverse effects. ${ }^{\mathbf{1 , 2}}$ As an important EDC, estrogens share the characteristics of EDCs, such as they may interact with the endocrine system of organisms and cause non-negligible adverse effects on the reproduction of wildlife and humans. ${ }^{3-5}$ One such example of an EDC is $17 \beta$-estradiol-3benzoate (E2B) which is a ubiquitous environmental agent. It is an estradiol analog commonly used in livestock for inducing weight gain and synchronising estrus cycles in heifers and cattle. ${ }^{6,7}$ However, as an analogue of EDC, E2B binds with estrogen receptors (ERs) which are expressed in human cells such as breast, bone and prostate, leading to relevant metastatic tumour formation. ${ }^{8,9}$ As early as 1988, the use of this kind of xenobiotic agent for improving the growth and feed conversion rates of food-producing animals has been banned in the European Union (EU) and no residues of these anabolic substances should be present in animal products. ${ }^{7}$ To enforce the prohibition on anabolic steroid abuse and prevent the illegal use of E2B, effective monitoring and detection methods have been developed.

For E2B determination, the most common techniques in use are high-performance liquid chromatography (HPLC), gas chromatography (GC), ${ }^{\mathbf{1 0 - 1 2}}$ gas chromatography-mass spectrometry (GC-MS), ${ }^{13}$ radioimmunoassay, ${ }^{14}$ and liquid

${ }^{a}$ College of Science, Huazhong Agricultural University, Wuhan 430070, P. R. China. E-mail: zhangying84@mail.hzau.edu.cn

${ }^{b}$ Faculty of Science and Engineering, Manchester Metropolitan University, Chester Street, Manchester M1 5GD, UK. E-mail: c.banks@mmu.ac.uk

$\dagger$ Electronic supplementary information (ESI) available. See DOI: 10.1039/c7ay01790a
Although electrochemical methods have been demonstrated to exhibit many advantages over the traditional analytical methods, including rapid analysis, economical instrumentation, simple operation and high sensitivity and excellent selectivity, there are, to the best of our knowledge, no reports on the electrochemical determination of this specific endocrine disruptor.

In our previous research, hydroxyapatite $\left(\mathrm{Ca}_{5}\left(\mathrm{PO}_{4}\right)_{3} \mathrm{OH}, \mathrm{HAp}\right)$ fabricated by template-assisted biomineralisation has been extensively applied in the field of chemically modified electrodes for biosensors, mainly due to its high biocompatibility and unique three-dimensional network structure. ${ }^{\text {16-21 }}$ HAp materials with a hierarchical structure originating from a natural egg-shell have been successfully applied in the electrochemical sensing of bisphenol A (BPA) with a detection limit of $2.46 \mathrm{nM} .{ }^{18}$ On the other hand, it has been demonstrated that HAp materials manipulated by cation-exchange membraneassisted biomineralisation have significant electrochemical sensing capabilities attributed to their three-dimensional networks and beneficial electron transport process. ${ }^{\mathbf{1 6}}$ In this work, we demonstrate proof-of-concept for the electroanalytical sensing of E2B using hydroxyapatite, which gives rise to highly sensitive analytical performances within aqueous solutions due to the material's inherent three-dimensional channel frameworks and useful electron transport capabilities.

\section{Results and discussion}

The fabrication of the hydroxyapatite (HAp) material/surface was performed as described in our previous report, ${ }^{16}$ and for readers' convenience, these details are reported in the ESI. $\dagger$ In brief, the growth of HAp is performed in a self-designed crystallizer (Fig. $\mathrm{S} 1 \dagger$ ) at a $\mathrm{pH}$ of 11 in order to ensure the orientational growth of HAp crystals. After holding for 1 to 7 days, the crystals 
are scraped off the membrane and constructed into chemically modified carbon paste electrodes, denoted throughout as HApCPE. The crystalline phase and structure evolution during the 1 to 7 days' fabrication process (see the ESI $\dagger$ ) are illustrated in Fig. 1. The corresponding PDF numbers are indicated along with the X-ray diffraction (XRD) patterns. Initially, the crystals are mainly composed of monetite $\left(\mathrm{CaPO}_{3} \mathrm{OH}\right.$, PDF 09-0080) after 1 day. After extending the time of reaction from 3 to 7 days, the major constituents of the material are transformed to HAp with the variety of crystalline parameters such as the interplanar spacing $(d)$ values of the strongest three diffraction peaks of the (211), (112) and (002) planes and lattice constants in each sample calculated by peak profile and cell refinement in order to reflect the transformation tendency, as shown in Table $\mathrm{S} 1 . \dagger$ The values of $d_{002}, d_{112}$ and $c$ increase with the prolonged reaction time, while the others display a decrease. The variation of the unit cell for each crystalline structure with time is schematically shown in Fig. 2. The HAp structured family, which commonly display a $P 6_{3} / m$ hexagonal symmetry and have the ideal crystallochemical formula $\mathrm{Ca}_{10}\left(\mathrm{PO}_{4}\right)_{6}(\mathrm{OH})_{2}$, can be described as onedimensional ion conductors, where the $\left[\mathrm{Ca}_{4}^{\mathrm{I}}\right]\left[\left(\mathrm{PO}_{4}\right)_{6}\right]$ framework is constructed of face-sharing $\mathrm{Ca}^{\mathrm{I}} \mathrm{O}_{6}$ metaprism columns linked

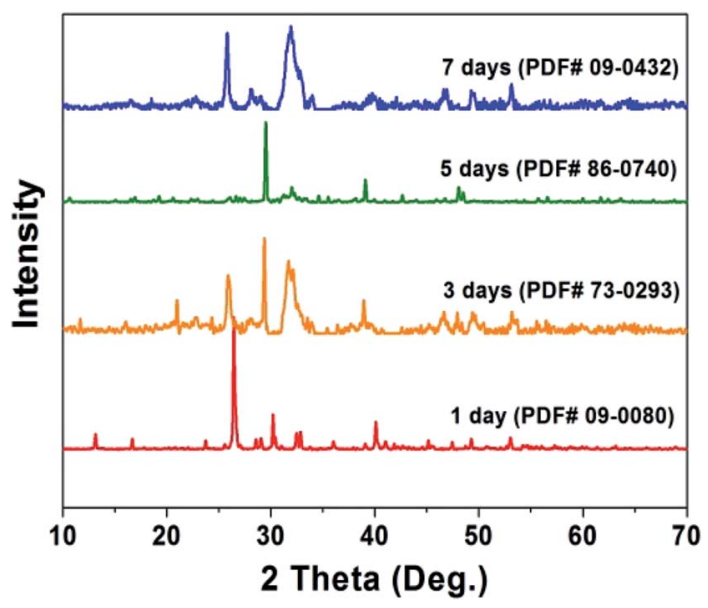

Fig. 1 X-ray diffraction patterns of the crystals after 1 to 7 days' fabrication time. through corner-connection to isolated $\mathrm{PO}_{4}$ tetrahedra. The resulting channels contain $\mathrm{Ca}^{\mathrm{II}}$ and $\mathrm{OH}$ ions that are potentially ion conducting and exchangeable and the ion conduction is essentially electron transportation. It is likely that the variation of these lattice parameters will impact the sensing capability of the HAp material towards E2B; a suitable crystalline lattice will potentially favour electron transfer and provide a useful electrochemical surface.

The electrochemical behaviour of E2B at the HAp-CPE in different methanol : PBS ratios is illustrated in Fig. 3. It is readily evident that there is a clear electrochemical reduction peak facilitated by the HAp. Note that the response of the HAp-CPE in the presence and absence of E2B is presented in Fig. $\mathrm{S} 2 \uparrow$ indicating that the voltammetric signal is due to the electrochemical reduction of E2B and not any other electrochemical processes such as the supporting electrolyte degradation. The effect of changing the voltammetric scan rate is shown in Fig. 3 where the peak potential shifts with the scan rate and the shape of the voltammetric signatures indicates an electrochemically irreversible reaction. The underlying electrochemical process is likely the electrochemical reduction of the $-\mathrm{C}=\mathrm{O}$ group to $-\mathrm{C}-$ $\mathrm{OH}$ via a one-electron and one-proton process. Analysis of the current as a function of the scan rate and square-root of scan and the peak potential $\left(E_{\mathrm{r}}\right)$ as a function of natural logarithm of scan rate $(\ln v)$ are presented in Fig. 3. In the case of using an electrolyte/solution of methanol : PBS ratio of $10: 90$, the peak current is found to be linearly dependent on the scan rate indicating that the electrochemical process is adsorptioncontrolled, while in the case of the methanol : PBS ratio of $90: 10$, the peak current is linear in the case of the square-root of scan rate indicating a diffusion-controlled process; clearly, the solution/electrolyte has a key influence on the electrochemical process. Moreover, it was obtained that in both the mixtures, the natural logarithm of scan rate $(\ln v)$ was linearly related to the peak potential $\left(E_{\mathrm{r}}\right)$. Further analysis of the voltammetric data presented in Fig. 3 in the form of a $\log I p$ vs. $\log$ scan rate produces a slope of 0.85 indicating a mixed adsorption and diffusional response in the case of the methanol : PBS ratio of $90: 10$. The electron transfer number $(n)$ was deduced for the electrochemical reduction of E2B using the HAp-CPE and is presented in the ESI. $\dagger$

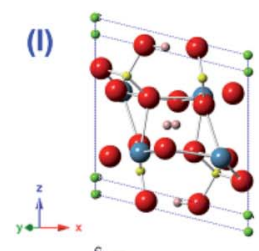

(I)

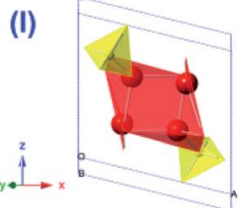

(II)

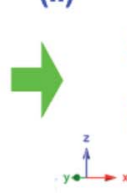

(II)

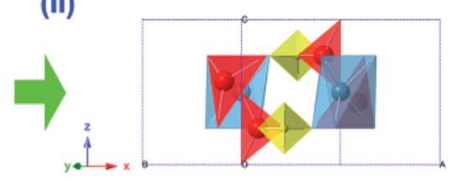

(III)

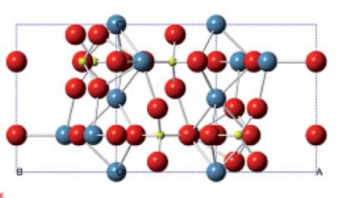

(III)

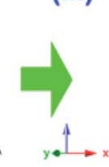

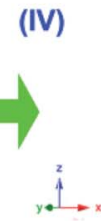

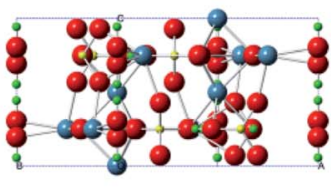

(IV)

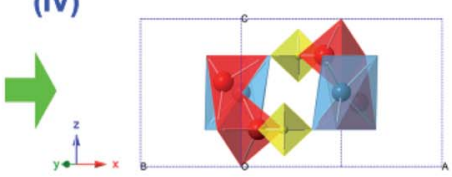

Fig. 2 Ball-stick models and polyhedral representations of crystalline structure evolution after (I) 1 day, (II) 3 days, (III) 5 days and (IV) 7 days' fabrications. All the angles of view are in the [010] plane (blue ball: Ca, red ball: O, yellow ball: P, green ball: $\mathrm{H}$ ). 

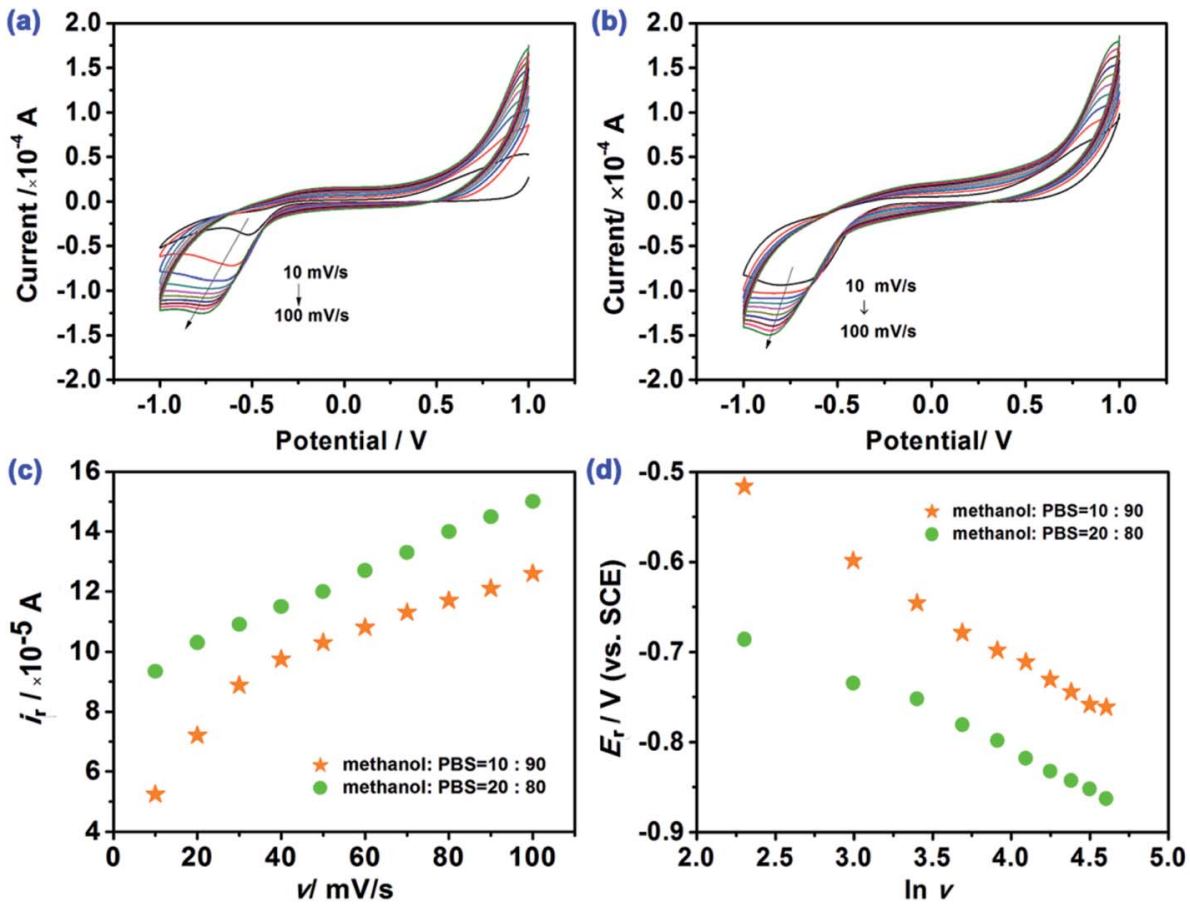

Fig. 3 Cyclic voltammetric responses of $1 \mu \mathrm{g} \mathrm{mL}^{-1} \mathrm{E} 2 \mathrm{~B}$ using the HAp-CPE modified with $40 \%$ of 7 day HAp over the range of scan rates from $10 \mathrm{mV} \mathrm{s}^{-1}$ to $100 \mathrm{mV} \mathrm{s}^{-1}$ in methanol: PBS = (a) $10: 90(\mathrm{v} / \mathrm{v})$ and (b) $90: 10(\mathrm{v} / \mathrm{v})$. (c) Dependence of the reduction peak current $\left(i_{\mathrm{r}}\right)$ on the voltammetric scan rate $(v)$. (d) The relationship between the reduction potential $\left(E_{r}\right)$ and natural logarithm of voltammetric scan rate (ln $\left.v\right)$
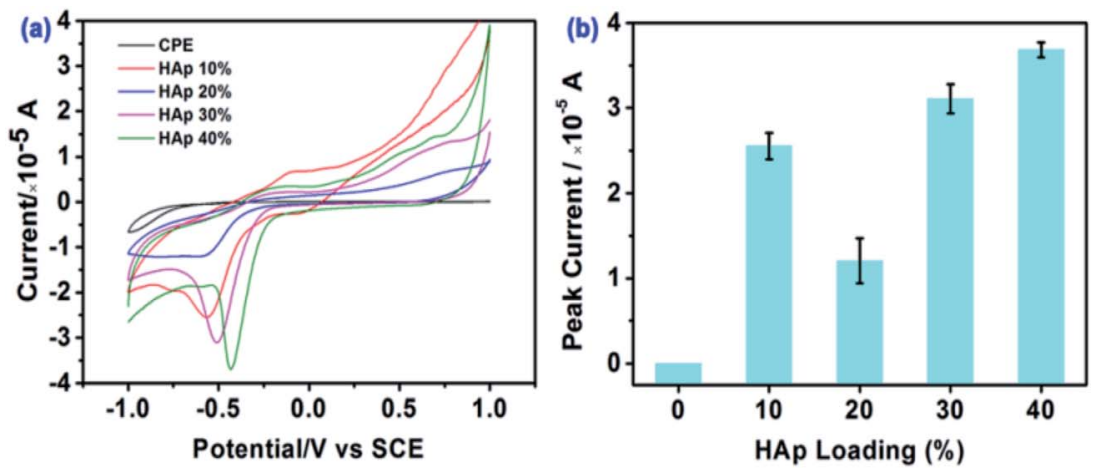

Fig. 4 Cyclic voltammetric curves of the 7 day HAp modified electrode in methanol : PBS = $10: 90$ (v/v) using different HAp loadings. E2B concentration: $1 \mu \mathrm{g} \mathrm{mL}^{-1}$

The effect of reaction time (days) on HAp utilised within the HAp-CPE was also explored towards the magnitude of the electrochemical signal corresponding to the electrochemical reduction of $\mathrm{E} 2 \mathrm{~B}$. It was found that the peak current corresponded to $0.6 \times 10^{-5} \mathrm{~A}$ for 1 day fabrication time where the magnitude of the peak current was found to increase to $1.1 \times 10^{-5} \mathrm{~A}$ for 7 days' fabrication time; consequently, the latter was chosen for further experiments. Next, loading (\%) with the HAp-CPE was explored with the voltammetric signal monitored as a function of HAp loading from 10 to $40 \%$. Fig. 4 shows the voltammetric signatures along with the effect on the peak current/signal. It is interesting to note that the voltammetric peak current shifts to less positive potentials as the \% of HAp is increased along with a corresponding increase in the peak height with the exception of the $20 \%$ HAp loading. The shape of the cyclic voltammogram also quantitatively changes from that of being largely diffusional to that of a thin-layer type response, which might be incorrectly assumed to be a transition to an electrocatalytic surface rather than true changes in the surface morphology and associated mass transport. Consequently, the largest voltammetric response/peak height is observed with a $40 \%$ HAp loading which is a combination of a useful electrochemical material being introduced into the electrode along with changes in the voltammetric response from having a contribution from a mixed diffusion and an adsorption electrochemical process (see earlier)/thin-layer voltammetry.

The analytical performance of the HAp-CPE towards the sensing of E2B was evaluated using cyclic voltammetry. Additions 

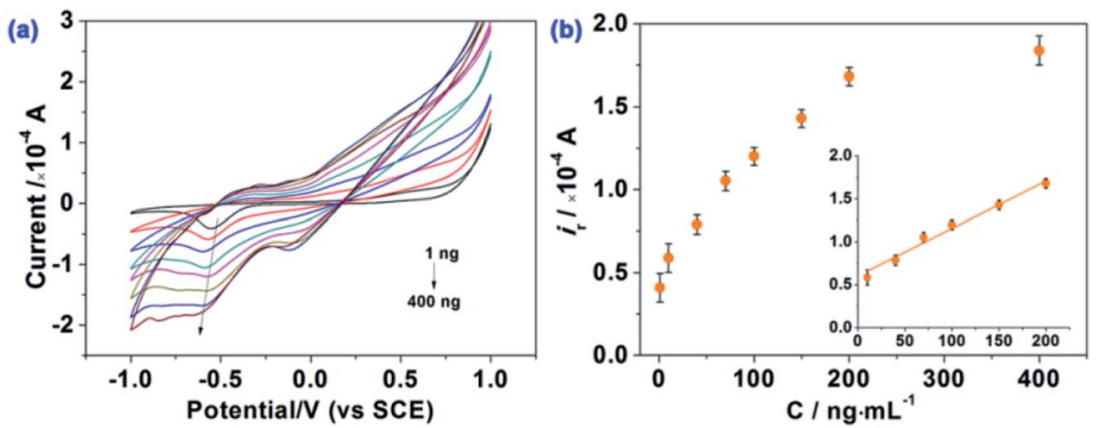

Fig. 5 Typical cyclic voltammetric responses and associated calibration plot obtained using the HAp-CPE towards the sensing of E2B. Solution composition; methanol: PBS =10: 90 (v/v). HAp: 7 day fabrication time; 40\% loading of HAp in the HAp-CPE. Concentration of E2B: 1 ng mL ${ }^{-1}$ $\sim 400 \mathrm{ng} \mathrm{mL}^{-1}$. Error bars are an average of three measurements and their associated standard deviation.

of E2B over the range of $1 \mathrm{ng} \mathrm{mL}^{-1}$ to $400 \mathrm{ng} \mathrm{mL} \mathrm{m}^{-1}$ were made into a methanol $: \mathrm{PBS}=20: 80(\mathrm{v} / \mathrm{v})$ solution as shown in Fig. 5 . It is evident that at low E2B concentrations a single reduction wave is observed where at higher concentrations a pre-wave appears reflecting the adsorption of E2B on the HAp surface within the HAp-CPE. Analysis of the voltammetric response as a function of concentration, as shown in Fig. 5, shows a linear response at low E2B concentrations, which is observed to plateau at higher concentrations due to the saturation of E2B on the HAp surface. Analysis of the linear portion of the calibration plot reveals a linear response over from 1 to $200 \mathrm{ng} \mathrm{mL}^{-1}\left(i_{\mathrm{p}}\left(\times 10^{-4} \mathrm{~A}\right)=\right.$ $\left.0.00551 C_{\mathrm{E} 2 \mathrm{~B}}\left(\mathrm{ng} \mathrm{mL}{ }^{-1}\right)+0.60681 ; R^{2}=0.98\right)$. Based on this linear range, the detection limit (based on $3 \sigma$ ) was determined to correspond to $24.4 \mathrm{ng} \mathrm{L}^{-1}(0.065 \mathrm{nM})$, which is much lower than the previous reports for the determination/sensing of E2B using GC $\left(0.15 \mathrm{mg} \mathrm{L}^{-1}\right)^{12}$ and is comparable with GC-MS/MS or LC-MS/ MS $\left(4.1 \mu \mathrm{g} \mathrm{kg}{ }^{-1}\right),{ }^{7}$ indicating that this proposed method can potentially be used for the sensitive monitoring of low E2B concentrations. Note that this is the first reported example of the electrochemical sensing of E2B and hence no comparison to other electroanalytical methodologies can be made.

\section{Conclusions}

In summary, we have demonstrated a proof-of-concept for the electrochemical sensing method of the pollutant E2B within methanol/PBS mixtures. Sensitive analytical performances are observed using HAp as the basis of the electrochemical sensing platform due to its favourable electrochemical response and 3D framework with a possible detection limit of $24.4 \mathrm{ng} \mathrm{L}^{-1}(0.065$ $\mathrm{nM})$. Future work is recommended for exploring this material in real applications.

\section{Conflicts of interest}

There are no conflicts to declare.

\section{Acknowledgements}

This work was financially supported by the National Undergraduate Training Program for Innovation and
Entrepreneurship (No. 201710504090), Students Research Fund of Huazhong Agricultural University (SRF, No. 2017357) and National Natural Science Foundation of China (No. 31500803).

\section{References}

1 E. Kopras, V. Potluri, M.-L. Bermudez, K. Williams, S. Belcher and S. Kasper, Actions of endocrine-disrupting chemicals on stem/progenitor cells during development and disease, Endocr-Relat. Cancer, 2014, 21(2), T1-T12.

2 J. Ochieng, G. N. Nangami, O. Ogunkua, I. R. Miousse, I. Koturbash, V. Odero-Marah, L. J. McCawley, P. NangiaMakker, N. Ahmed, Y. Luqmani, Z. Chen, S. Papagerakis, G. T. Wolf, C. Dong, B. P. Zhou, D. G. Brown, A. M. Colacci, R. A. Hamid, C. Mondello, J. Raju, E. P. Ryan, J. Woodrick, A. I. Scovassi, N. Singh, M. Vaccari, R. Roy, S. Forte, L. Memeo, H. K. Salem, A. Amedei, R. AlTemaimi, F. Al-Mulla, W. H. Bisson and S. E. Eltom, The impact of low-dose carcinogens and environmental disruptors on tissue invasion and metastasis, Carcinogenesis, 2015, 36(suppl. 1), S128-S159.

3 W.-Y. Hu, G.-B. Shi, D.-P. Hu, J. L. Nelles and G. S. Prins, Actions of estrogens and endocrine disrupting chemicals on human prostate stem/progenitor cells and prostate cancer risk, Mol. Cell. Endocrinol., 2012, 354(1-2), 63-73.

4 Q. Wang, K. Rangiah, C. Mesaros, N. W. Snyder, A. Vachani, H. Song and I. A. Blair, Ultrasensitive quantification of serum estrogens in postmenopausal women and older men by liquid chromatography-tandem mass spectrometry, Steroids, 2015, 96, 140-152.

5 J. P. Laurenson, R. A. Bloom, S. Page and N. Sadrieh, Ethinyl Estradiol and Other Human Pharmaceutical Estrogens in the Aquatic Environment: A Review of Recent Risk Assessment Data, AAPS J., 2014, 16(2), 299-310.

6 E. S. M. Silva, J. F. Roser, A. R. C. Gomes, S. C. Fritsch, J. C. F. Pantoja, J. P. Oliveira-Filho and C. Meira, Comparison of different regimens of estradiol benzoate treatments followed by long-acting progesterone to prepare noncycling mares as embryo recipients, Theriogenology, 2016, 86(7), 1749-1756. 
7 L. Rambaud, E. Bichon, N. Cesbron, F. André and B. L. Bizec, Study of $17 \beta$-estradiol-3-benzoate, $17 \alpha$-methyltestosterone and medroxyprogesterone acetate fixation in bovine hair, Anal. Chim. Acta, 2005, 532(2), 165-176.

8 K. Maruyama, M. Nakamura, S. Tomoshige, K. Sugita, M. Makishima, Y. Hashimoto and M. Ishikawa, Structureactivity relationships of bisphenol A analogs at estrogen receptors (ERs): Discovery of an ER $\alpha$-selective antagonist, Bioorg. Med. Chem. Lett., 2013, 23(14), 4031-4036.

9 E. Di Zazzo, G. Galasso, P. Giovannelli, M. Di Donato, A. Di Santi, G. Cernera, V. Rossi, C. Abbondanza, B. Moncharmont, A. A. Sinisi, G. Castoria and A. Migliaccio, Prostate cancer stem cells: the role of androgen and estrogen receptors, Oncotarget, 2016, 7(1), 193-208.

10 H. Noppe, B. Le Bizec, K. Verheyden and H. F. De Brabander, Novel analytical methods for the determination of steroid hormones in edible matrices, Anal. Chim. Acta, 2008, 611(1), 1-16.

11 G. Pinel, L. Rambaud, F. Monteau, C. Elliot and B. Le Bizec, Estranediols profiling in calves' urine after $17 \beta$-nandrolone laureate ester administration, J. Steroid Biochem. Mol. Biol., 2010, 121(3), 626-632.

12 G. Pinel, L. Rambaud, G. Cacciatore, A. Bergwerff, C. Elliott, M. Nielen and B. Le Bizec, Elimination kinetic of $17 \beta$ estradiol 3-benzoate and $17 \beta$-nandrolone laureate ester metabolites in calves' urine, J. Steroid Biochem. Mol. Biol., 2008, 110(1), 30-38.

13 D. Thieme, P. Anielski, J. Grosse, H. Sachs and R. K. Mueller, Identification of anabolic steroids in serum, urine, sweat and hair, Anal. Chim. Acta, 2003, 483(1), 299-306.

14 P. Loutchanwoot, T. Vortherms and H. Jarry, Evaluation of in vivo estrogenic potency of natural estrogen-active chemical, puerarin, on pituitary function in gonadectomized female rats, Life Sci., 2016, 165, 75-82.

15 E. Duffy, M. H. Mooney, C. T. Elliott and M. O'Keeffe, Studies on the persistence of estradiol benzoate and nortestosterone decanoate in hair of cattle following treatment with growth promoters, determined by ultra-high-performance liquid chromatography-tandem mass spectrometry, J. Chromatogr. A, 2009, 1216(46), 8090-8095.

16 Y. Zhang, Y. Liu, X. Ji, C. E. Banks and W. Zhang, Sea cucumber-like hydroxyapatite: cation exchange membraneassisted synthesis and its application in ultra-sensitive heavy metal detection, Chem. Commun., 2011, 47(14), 41264128.

17 Y. Zhang, Y. Liu, X. Ji, C. E. Banks and W. Zhang, Flower-like hydroxyapatite modified carbon paste electrodes applicable for highly sensitive detection of heavy metal ions, J. Mater. Chem., 2011, 21(21), 7552.

18 Y. Zhang, Y. Liu, X. Ji, C. E. Banks and W. Zhang, Conversion of egg-shell to hydroxyapatite for highly sensitive detection of endocrine disruptor bisphenol A, J. Mater. Chem., 2011, 21(38), 14428.

19 Y. Zhang, Y. Liu, X. Ji, C. E. Banks and W. Zhang, Conversion of natural egg-shell to 3D flower-like hydroxyapatite agglomerates for highly sensitive detection of As3+ ions, Mater. Lett., 2012, 78, 120-123.

20 Y. Zhang, W. Zhang, Q. Zhang, K. Li, W. Liu, Y. Liu and C. E. Banks, Green electrochemical sensing platforms: utilizing hydroxyapatite derived from natural fish scales as a novel electrochemical material for the sensitive detection of kidney injury molecule 1 (KIM-1), Analyst, 2014, 139(21), 5362-5366.

21 S. Dong, G. Che and Y. Xie, Chemical Modified Electrodes, Science Press Co. Ltd., Beijing, 2003. 